\title{
Performance Analysis for Dual Band PCS Networks
}

\author{
Yi-Bing Lin, Senior Member, IEEE, Wei-Ru Lai, and Rong-Jaye Chen, Member, IEEE
}

\begin{abstract}
In a dual band personal communications services (PCS) network, heavy traffic areas are covered by microcells which overlay macrocells. In such a network, microcells and macrocells utilize different frequency bands. We propose an analytic model to study the performance of a dual band PCS architecture. Our model assumes that a PCS subscriber has a general residence time distribution in a microcell and the macrocell residence time distribution is derived from the microcell residence time distribution. An iterative algorithm is used to compute the overflow traffic from a microcell to its overlaid macrocell. Then, the call incompletion probability is computed by a macrocell model based on the overflow traffic. Our study indicates that the variance of the microcell residence time distribution and the number of microcells covered by a macrocell have significant effects on the call incompletion probability.
\end{abstract}

Index Terms—Dual band, GSM, handoff, macrocell, microcell, personal communications services.

\section{INTRODUCTION}

A PERSONAL Communications services (PCS) network provides communication services to mobile users via their mobile stations (MS) or handsets. When the PCS network attempts to deliver a call to an MS or the MS attempts to originate a call, the call is connected between the MS and the base transceiver station (BTS) through the radio channel within the radio coverage of the BTS or the cell. If no idle radio channel is available, the call is blocked. When the MS moves from a cell to another during the conversation, a handoff occurs. To continue the call, the MS requests an idle radio channel in the new cell to set up a new connection and drops the connection to the old cell. If the handoff is rejected because no idle radio channel is available in the new cell, the call is forced to terminate. PCS networks such as GSM [16] exercise the nonprioritized channel allocation scheme in which the BTS handles a handoff request exactly the same as a new call. In a PCS network, an important performance measure is the incompletion probability or the probability that a call is blocked or forced to terminate.

PCS channel assignment has been intensively studied in the literature [3], [4], [6], [10], [17], [21]. In heavy traffic areas, the new call blocking probability and the forced termination probability grow rapidly as traffic increases. Microcell/macrocell architectures have been proposed [2], [18], [8], [7] to reduce the call incompletion probability. In Taiwan, a dual band GSM microcell/macrocell cellular network has been deployed by Far EasTone Telecommunications Co., Ltd. to improve the capacity of GSM services

- Y.-B. Lin and R.-J. Chen are with the Department of Computer Science and Information Engineering, National Chaio Tung University, Hsinchu, Taiwan, ROC. E-mail: \{liny, rjchen\}@csie.nctu.edu.tw.

- W.-R. Lai is with the Department of Information Management, Chin-Min College, Tou-Fen, Miao-Li, Taiwan, ROC.

E-mail: wrlai@mis.chinmin.edu.tw.

Manuscript accepted 24 Nov. 1999.

For information on obtaining reprints of this article, please send e-mail to: tc@computer.org, and reference IEEECS Log Number 108280.
[16]. This microcell/macrocell configuration (see Fig. 1) consists of two types of BTSs: The DCS1800 BTSs operate at $1.8 \mathrm{GHz}$ and the GSM900 BTSs operate at $900 \mathrm{MHz}$. In this configuration, the GSM900 BTSs serve as the base stations for macrocells while the DCS1800 BTSs serve as the microcell base stations. Every macrocell overlays with one or more microcells (see Fig. 2a). A GSM900 BTS and its overlaid DCS1800 BTSs are connected to the same Base Station Controller (BSC), as illustrated in Fig. 1. The BSCs connect to mobile switching centers (MSCs) and the MSCs connect to the switches in the public switched telephone network. The reader is referred to [16] for the details of BSC and MSC.

In this dual band configuration, a new call arriving at the system is first handled by a microcell. If no channel is available in the microcell, the call is handled by the macrocell. The call is blocked if no channel is available in the macrocell (see Fig. 2b). During the conversation, an MS may move from a microcell to another microcell and the call will be handed over among microcells. During a microcell handoff, if no channel is available in the new microcell, the handoff call is handled by the macrocell. In a dual band network, it is important to determine the number of microcells covered by a macrocell to achieve good performance (i.e., low call incompletion probability). This paper studies how the microcell/macrocell configuration and the MS movement behavior affect the dual band network performance. We first derive the MS residence time distribution (the interval between the arrival and the departure of an MS in a cell) for a macrocell based on the microcell residence time distribution. By using the overflow traffic as the input, the call incompletion probability is computed in the macrocell model (see Fig. 2b).

\section{The Microcell Model}

The microcell model to be presented in this section generalizes our previous work [14]. Three assumptions are used in this paper: 


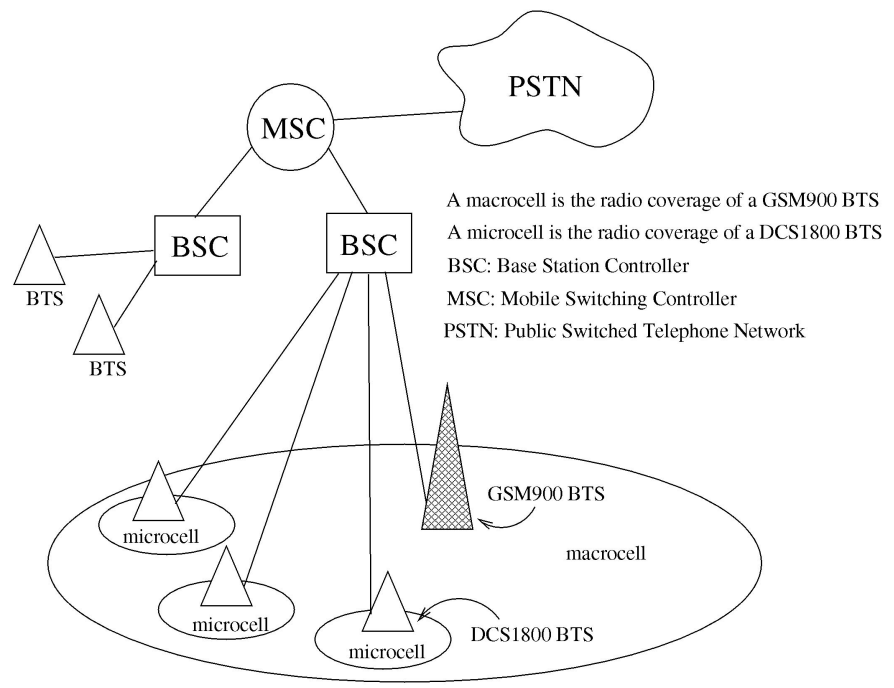

Fig. 1. Dual band GSM network.

Assumption 1. The phone call arrivals to an MS are a Poisson process.

Assumption 2. The call holding time (the interval between the time the conversation starts and the time it completes) is exponentially distributed.

Assumption 3. The microcell residence times of an MS have a general distribution.

Assumption $\mathbf{1}$ is required to derive the interval between the time of a call arrival and when the MS leaves the microcell (to be elaborated in Section 2.1). This assumption also guarantees that the net call arrival process to a microcell is
Poisson. Note that, as long as the number of MS in a microcell is sufficiently large, the net call arrivals to a microcell are Poisson even if the call arrivals to individual MS are not. Assumption 2 does not hold for wireline calls. Many telephone companies offer flat rate charging for local phone calls and, so, very long call holding times are observed (e.g., computer dial-up connection for several days). Thus, long tails may be expected in the call holding time distributions. In this case, Lognormal or Gamma distributions are more appropriate to approximate the call holding time distribution. Cellular phone calls, on the other hand, are charged based on the air-time usage, and the

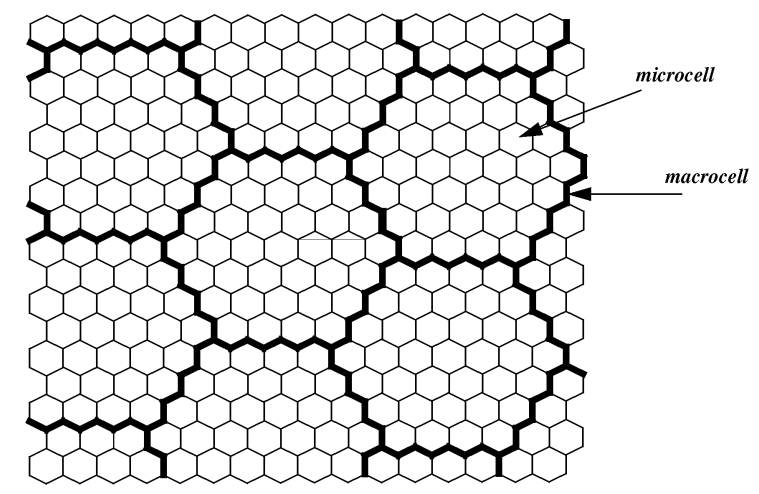

(a)

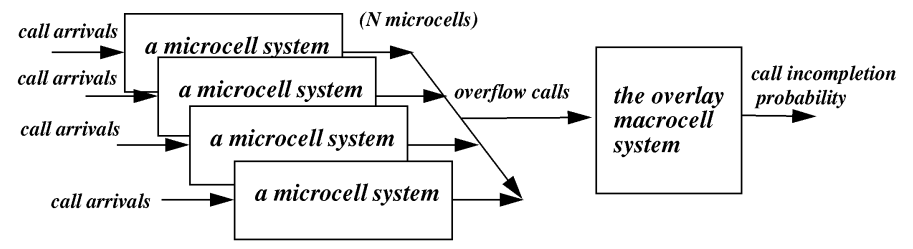

(b)

Fig. 2. Homogeneous microcell/macrocell layout and channel assignment procedure. (a) Microcell/macrocell configuration. (b) The channel assignment procedure. 


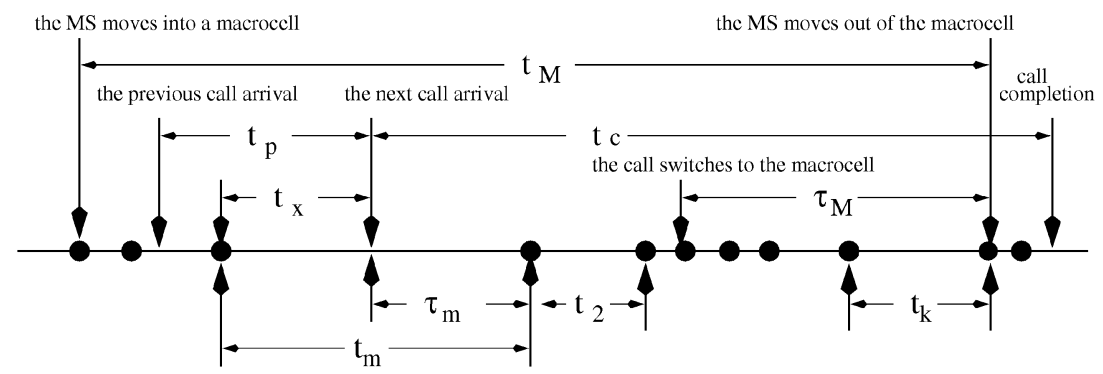

the MS moves into a microcell

the MS moves out of the microcell

Fig. 3. The timing diagram.

users do not hold unnecessary long calls. Data measured from real GSM network operation indicated that exponential call holding time distribution is reasonable and the mean call holding time is typically less than one third of that for wireline calls.

\subsection{The MS Residence Time Distributions}

Consider the timing diagram in Fig. 3. From Assumption 1, the call arrivals to an MS are a Poisson process with arrival rate $\lambda$. That is, $t_{p}$ has a density function $\lambda e^{-\lambda t_{p}}$. From Assumption 3, the MS residence time $t_{m}$ at microcell $C_{i}$ has a general density function $f_{m, i}\left(t_{m}\right)$ and the distribution $F_{m, i}\left(t_{m}\right)=\int_{\tau=0}^{t_{m}} f_{m, i}(\tau) d \tau$. Let $\tau_{m}$ be the interval between when a call arrives and when the MS moves out of $C_{i}$. Let $r_{m, i}\left(\tau_{m}\right)$ be the density function of $\tau_{m}$. If $f_{m, i}(t)=f_{m, j}(t)$ for all $i, j$, then $r_{m, i}(t)=r_{m, j}(t)$ can be derived by using the excess life property [5]. Unfortunately, the excess life property does not apply when $f_{m, i}(t) \neq f_{m, j}(t)$ and the residence time distribution should be derived as follows: From the definition of density function, we have

$$
r_{m, i}\left(\tau_{m}\right)=\lim _{d \tau_{m} \rightarrow 0} \frac{\operatorname{Pr}\left[\tau_{m} d \tau_{m} \text { where } \tau_{m}=t_{m}-t_{x} \mid t_{m}>t_{x}\right]}{d \tau_{m}} .
$$

From Assumption 1 and the memoryless property of the exponential distribution, $t_{x}$ has the same exponential distribution as $t_{p}$ (where $t_{x}$ and $t_{p}$ are illustrated in Fig. 3). Thus, (1) is rewritten as

$$
\begin{aligned}
r_{m, i}\left(\tau_{m}\right) & =\lim _{d \tau_{m} \rightarrow 0} \frac{\operatorname{Pr}\left[\tau_{m} d \tau_{m} \text { where } \tau_{m}=t_{m}-t_{x} \text { and } t_{m}>t_{x}\right]}{d \tau_{m}} \\
& =\int_{t_{x}=0}^{\infty} \lambda e^{-\lambda t_{x}} f_{m, i}\left(t_{x}+\tau_{m}\right) d t_{x} \\
& =\lambda e^{\lambda \tau_{m}}\left[f_{\mathbf{m}, i}^{*}(\lambda)-\int_{t^{\prime}=0}^{\tau_{m}} f_{m, i}\left(t^{\prime}\right) e^{-\lambda t^{\prime}} d t^{\prime}\right]
\end{aligned}
$$

Equation (3) is derived from (2) by using the Laplace transform rule P5.2.1 in [19], where

$$
f_{\mathbf{m}, i}^{*}(s)=\int_{\tau_{m}=0}^{\infty} f_{m, i}\left(\tau_{m}\right) e^{-s \tau_{m}} d \tau_{m}
$$

is the Laplace transform for the MS residence time distribution $F_{m, i}\left(\tau_{m}\right)$. The probability $\operatorname{Pr}\left[t_{m}>t_{x}\right]$ is

$$
\begin{aligned}
\operatorname{Pr}\left[t_{m}>t_{x}\right] & =\int_{t_{m}=0}^{\infty} \int_{t_{x}=0}^{t_{m}} f_{m, i}\left(t_{m}\right) \lambda e^{-\lambda t_{x}} d t_{x} d t_{m} \\
& =1-f_{\mathbf{m}, i}^{*}(\lambda) .
\end{aligned}
$$

From (3) and (4) and since

$$
\operatorname{Pr}\left[\tau_{m}=t_{m}-t_{x} \mid t_{m}>t_{x}\right]=\frac{\operatorname{Pr}\left[\tau_{m}=t_{m}-t_{x} \text { and } t_{m}>t_{x}\right]}{\operatorname{Pr}\left[t_{m}>t_{x}\right]},
$$

we have

$$
r_{m, i}\left(\tau_{m}\right)=\frac{\lambda e^{\lambda \tau_{m}}\left[f_{\mathbf{m}, i}^{*}(\lambda)-\int_{t^{\prime}=0}^{\tau_{m}} f_{m, i}\left(t^{\prime}\right) e^{-\lambda t^{\prime}} d t^{\prime}\right]}{1-f_{\mathbf{m}, i}^{*}(\lambda)}
$$

and its Laplace transform is

$$
\begin{aligned}
& r_{\mathbf{m}, i}^{*}(s)=\int_{\tau_{m}=0}^{\infty} r_{m, i}\left(\tau_{m}\right) e^{-s \tau_{m}} d \tau_{m} \\
& =\frac{\lambda\left[\int_{\tau_{m}=0}^{\infty} f_{m, i}^{*}(\lambda) e^{\lambda \tau_{m}} e^{-s \tau_{m}} d \tau_{m}-\int_{\tau_{m}=0}^{\infty} e^{(\lambda-s) \tau_{m}} \int_{t^{\prime}=0}^{\tau_{m}} f_{m, i}\left(\tau_{m}\right) e^{-\lambda t^{\prime}} d t^{\prime} d \tau_{m}\right]}{1-f_{\mathbf{m}, i}^{*}(\lambda)} \\
& =\left\{\frac{\lambda}{(s-\lambda)\left[1-f_{\mathbf{m}, i}^{*}(\lambda)\right]}\right\}\left[f_{\mathbf{m}, i}^{*}(\lambda)-f_{\mathbf{m}, i}^{*}(s)\right] .
\end{aligned}
$$

The first term of (6) is derived from the first term of (5) by using the Laplace transform rule P.1.3 in [19]. The second term of (6) is derived from the second term of (5) by using the Laplace transform rules P1.1.3 and P1.1.6 in [19].

In a practical PCS environment, the number of MSs in a cell is expected to be much larger than the number of channels, and the traffic (the net call arrival rate) to the microcell is engineered at one or two percent blocking probability, which implies that the call arrival rate per MS $\lambda \rightarrow 0$ for a fixed net call arrival stream [20]. If $f_{m, i}(t)$ is a Gamma density function [9] with the shape parameter $\alpha_{i}$ and mean $1 / \eta_{i}$ (i.e., with the scale parameter $\alpha_{i} \eta_{i}$ ), then $f_{\mathbf{m}, i}^{*}(s)=\left(\frac{\alpha_{i} \eta_{i}}{s+\alpha_{i} \eta_{i}}\right)^{\alpha_{i}}$. By using the 1'Hospital's rule, we showed [14] that, for Gamma residence time distribution,

$$
\lim _{\lambda \rightarrow 0} r_{\mathbf{m}, i}^{*}(s)=\frac{\eta_{i}}{s}\left[1-f_{\mathbf{m}, i}^{*}(s)\right],
$$


which is the same as the $r_{\mathrm{m}}^{*}(s)$ function for the homogeneous PCS network (i.e., when $f_{m, i}(t)=f_{m, j}(t)=f_{m}(t)$ for $i \neq j$ ) [15]. In other words, (7) indicates that if the call activity per user is not heavy (but the net call traffic to a cell can be either light or heavy), then the $\tau_{m}$ distribution for the heterogeneous network can be approximated by the homogeneous network.

We are particularly interested in the Gamma distribution. It has been shown that the distribution of any positive random variable can be approximated by a mixture of Gamma distributions (see Lemma 3.9 in [11]). One may also measure the MS residence times in a real PCS network and the measured data can be approximated by a Gamma distribution as the input to our handoff model. In a heterogeneous PCS network, it suffices to use the Gamma distributions with different shape and scale parameters to represent different MS residence time distributions.

\subsection{The Handoff Traffic}

Consider a group of $M$ microcells, where every macrocell covers $N$ microcells (i.e., there are $M / N$ macrocells, where $M>>N)$. Let $p_{i, j}$ be the probability that an MS moves from microcell $C_{i}$ to microcell $C_{j}$ where $1 \leq i, j \leq M$. It is clear that $p_{i, i}=0$. The handoff call arrival rate $\lambda_{h}(i)$ to $C_{i}$ is derived as follows:

Assume that the new call blocking probability $p_{o}(i)$ and the forced termination probability $p_{f}(i)$ for a microcell $C_{i}$ are known. We will show how to compute $p_{o}(i)$ and $p_{f}(i)$ in Section 2.5 .

Let $p_{v o}(j)\left(p_{v h}(j)\right)$ be the probability that a new call (handoff call) is not complete before the MS moves out of the the microcell $C_{j}$. From Assumption 2, the call holding time is exponentially distributed with the density function $f_{c}\left(t_{c}\right)=\mu e^{-\mu t_{c}}$. Then,

$$
\begin{aligned}
p_{v o}(j) & =\int_{t=0}^{\infty} \int_{t_{c}=t}^{\infty} f_{c}\left(t_{c}\right) r_{m, j}(t) d t_{c} d t \\
& =r_{\mathbf{m}, j}^{*}(\mu) .
\end{aligned}
$$

For a handoff call, the time $t_{c}^{\prime}$ between the arrival time of the handoff call at $C_{j}$ and its completion time has the same density function as $f_{c}$ (the memoryless property of the exponential distribution). Similar to the derivation for (8), we have

$$
p_{v h}(j)=\int_{t=0}^{\infty} \int_{t_{c}^{\prime}=t}^{\infty} f_{c}\left(t_{c}^{\prime}\right) f_{m, j}(t) d t_{c}^{\prime} d t=f_{\mathbf{m}, j}^{*}(\mu) .
$$

The rate of the handoff calls moving out of $C_{j}$ consists of the rate of new calls and the accepted move-in handoff calls that are not complete before the MSs move out of cell $C_{j}$. Let $\lambda_{o}(j)$ be the rate of the new calls in cell $C_{j}$. Following the derivation in [13], this rate is

$$
\lambda_{o}(j)\left[1-p_{o}(j)\right] p_{v o}(j)+\lambda_{h}(j)\left[1-p_{f}(j)\right] p_{v h}(j) .
$$

From (8), (9), and (10), the rate of the handoff calls moving into $C_{i}$ is

$$
\begin{gathered}
\lambda_{h}(i)=\sum_{1 \leq j \leq M} p_{j, i}\left\{\lambda_{o}(j)\left[1-p_{o}(j)\right] r_{\mathbf{m}, j}^{*}(\mu)\right. \\
\left.+\lambda_{h}(j)\left[1-p_{f}(j)\right] f_{\mathbf{m}, j}^{*}(\mu)\right\} .
\end{gathered}
$$

For a homogeneous PCS network, we have

$$
f_{\mathbf{m}, i}^{*}(s)=f_{\mathbf{m}, j}^{*}(s)=f_{\mathbf{m}}^{*}(s), r_{\mathbf{m}, i}^{*}(s)=r_{\mathbf{m}, j}^{*}(s)=r_{\mathbf{m}}^{*}(s),
$$

and $\lambda_{o}(i)=\lambda_{o}(j)=\lambda_{o}$. Then,

$$
p_{o}(j)=p_{o}(i)=p_{o}, p_{f}(i)=p_{f}(j)=p_{f},
$$

$\lambda_{h}(i)=\lambda_{h}(j)=\lambda_{h}$, and (11) is rewritten as

$$
\begin{aligned}
\lambda_{h} & =\lambda_{o}\left(1-p_{o}\right) r_{\mathbf{m}}^{*}(\mu)+\lambda_{h}\left(1-p_{f}\right) f_{\mathbf{m}}^{*}(\mu) \\
& =\frac{\left(1-p_{o}\right) r_{\mathbf{m}}^{*}(\mu) \lambda_{o}}{1-\left(1-p_{f}\right) f_{\mathbf{m}}^{*}(\mu)},
\end{aligned}
$$

which is the the same as the $\lambda_{h}$ equation derived in [13].

\subsection{The Expected Channel Occupation Times}

When a channel of $C_{i}$ is assigned to a new call, the channel is released if the call completes or the MS moves out of the microcell. Let $t_{o n}(i)$ be the channel occupation time of a new call. Then,

$$
t_{\text {on }}(i)=\min \left(t_{c}, \tau_{m}\right) .
$$

For the example in Fig. $3, t_{o n}(i)=\tau_{m}$. The expected channel occupation time of a new call is derived as

$$
\begin{aligned}
E\left[t_{\text {on }}(i)\right] & =\int_{t_{c}=0}^{\infty} \int_{\tau_{m}=0}^{t_{c}} \tau_{m} r_{m, i}\left(\tau_{m}\right) \mu e^{-\mu t_{c}} d \tau_{m} d t_{c} \\
& +\int_{t_{c}=0}^{\infty} \int_{\tau_{m}=t_{c}}^{\infty} t_{c} r_{m, i}\left(\tau_{m}\right) \mu e^{-\mu t_{c}} d \tau_{m} d t_{c} \\
& =\frac{1}{\mu}\left[1-r_{\mathbf{m}, i}^{*}(\mu)\right] .
\end{aligned}
$$

Let $t_{o h}(i)$ be the channel occupation time of a handoff call. Similar to (13), the expected channel occupation time of a handoff call is

$$
E\left[t_{o h}(i)\right]=\frac{1}{\mu}\left[1-f_{\mathbf{m}, i}^{*}(\mu)\right] .
$$

Note that $E\left[t_{o h}(i)\right] \neq E\left[t_{o n}(i)\right]$ except for the case when $f_{m, i}(t)$ is an exponential density function.

\subsection{The Call Incompletion Probability}

A call is not complete if it is a blocked new call or a forcedterminated handoff call. Note that a call may make several successful handoffs before it is forced to terminate. Let $p_{n c}(i)$ be the incompletion probability of a (new) call initiated at $C_{i}$, and $\tilde{p}_{n c}(i)$ be the incompletion probability of a handoff call at $C_{i}$. Then, we have

$$
\begin{aligned}
& p_{n c}(i)=p_{o}(i)+\left[1-p_{o}(i)\right] p_{v o}(i)\left(\sum_{1 \leq j \leq M} p_{i, j} \tilde{p}_{n c}(j)\right) \\
& \tilde{p}_{n c}(i)=p_{f}(i)+\left[1-p_{f}(i)\right] p_{v h}(i)\left(\sum_{1 \leq j \leq M} p_{i, j} \tilde{p}_{n c}(j)\right) .
\end{aligned}
$$


Equation (15) implies that a new call at $C_{i}$ is incomplete if it is blocked at $C_{i}$ because no idle channel is available (with probability $p_{o}(i)$ ) or the call is connected (with probability $\left.1-p_{o}(i)\right)$, but the MS leaves $C_{i}$ before the call is complete (with probability $p_{v o}(i)$ ), and the call remains incomplete after the MS moves into a new microcell (with probability $\left.\sum_{1 \leq j \leq M} p_{i, j} \tilde{p}_{n c}(j)\right)$. Similarly, (16) implies that a handoff call at $C_{i}$ is not complete if no idle channel is available when the MS arrives at $C_{i}$ (with probability $p_{f}(i)$ ) or if the handoff call is accommodated at $C_{i}$ (with probability $\left.1-p_{f}(i)\right)$, but the MS leaves $C_{i}$ before the call is complete (with probability $p_{v h}(i)$ ), and the call is still not complete after the MS moves into a new microcell (with probability $\left.\sum_{1 \leq j \leq M} p_{i, j} \tilde{p}_{n c}(j)\right)$. In a homogeneous PCS network, $p_{n c}(i)=p_{n c}(j)=p_{n c}, \tilde{p}_{n c}(i)=\tilde{p}_{n c}(j)=\tilde{p}_{n c}$,

$$
\tilde{p}_{n c}=p_{f}+\left(1-p_{f}\right) p_{v h} \tilde{p}_{n c}=\frac{p_{f}}{1-\left(1-p_{f}\right) p_{v h}}
$$

and

$$
p_{n c}=p_{o}+\left(1-p_{o}\right) p_{v o} \tilde{p}_{n c}=p_{o}+\frac{\lambda_{h}}{\lambda_{o}} p_{f}
$$

\subsection{The Iterative Algorithm}

This section shows how to iteratively compute $p_{n c}(i)$ using the equations derived in the previous section. The iterative algorithm has the same steps as the homogeneous algorithm in [13] except that the equations used in every step are different. Consider a network of $M / N$ macrocells where every macrocell covers $N$ microcells (where $M>>N$ ).

\section{Algorithm 1 (Microcell).}

Input parameters: $p_{i, j}$ (the routing probability), $\lambda_{o}(i)$ (the new call arrival rate), $\mu$ (the call completion rate), $c_{i}$ (the number of channels in BTS $i$ ), $f_{m, i}(t)$ (the MS residence time density function), and $r_{m, i}(t)$, where $1 \leq i, j \leq M$.

Output measures: $\lambda_{h}(i)$ (the handoff call arrival rate), $p_{o}(i)$ (the new call blocking probability), $p_{f}(i)$ (the forced termination probability), and $p_{n c}(i)$ (the call incompletion probability), where $1 \leq i \leq M$.

Step 1. Select initial values for $\lambda_{h}(i)$ where $1 \leq i \leq M$.

Step 2. For $1 \leq i \leq M$, compute $p_{o}(i)$ and $p_{f}(i)$. In the nonprioritized scheme, the handoff calls and the new call attempts are not distinguishable, and $p_{o}(i)=p_{f}(i)$. In such a handoff scheme, channel allocation can be modeled after an $M / G / c / c$ queue [12] with the number of servers as $c_{i}$ and the job arrival rate as $\lambda_{o}(i)+\lambda_{h}(i)$ [13]. The load onto $C_{i}$ is computed using (13) and (14):

$$
\rho(i)=\lambda_{o}(i) E\left[t_{o n}(i)\right]+\lambda_{h}(i) E\left[t_{o h}(i)\right]
$$

and

$$
p_{f}(i)=p_{o}(i)=B\left(\rho(i), c_{i}\right)=\frac{\rho(i)^{c_{i}} / c_{i} !}{\sum_{j=0}^{c_{i}}\left(\rho(i)^{j} / j !\right)} .
$$

Step 3. For $1 \leq i \leq M, \lambda_{h, \text { old }}(i) \leftarrow \lambda_{h}(i)$.

Step 4. For $1 \leq i \leq M$, compute $\lambda_{h}(i)$ by using (11).

$$
\begin{gathered}
\lambda_{h}(i)=\sum_{1 \leq j \leq M} p_{j, i}\left\{\lambda_{o}(j)\left[1-p_{o}(j)\right] r_{\mathbf{m}, j}^{*}(\mu)\right. \\
\left.+\lambda_{h}(j)\left[1-p_{f}(j)\right] f_{\mathbf{m}, j}^{*}(\mu)\right\} .
\end{gathered}
$$

Step 5. If there exists $i$ such that $\left|\lambda_{h}(i)-\lambda_{h, o l d}(i)\right|>\delta \lambda_{h}(i)$, then go to Step 2. Otherwise, go to Step 6. Note that $\delta$ is a predefined value.

Step 6. Compute $p_{n c}(i)$ by using (8), (9), (15), and (16)

$$
\begin{aligned}
& p_{n c}(i)=p_{o}(i)+\left[1-p_{o}(i)\right] r_{\mathbf{m}, i}^{*}(\mu)\left(\sum_{1 \leq j \leq M} p_{i, j} \tilde{p}_{n c}(j)\right) \\
& \tilde{p}_{n c}(i)=p_{f}(i)+\left[1-p_{f}(i)\right] f_{\mathbf{m}, i}^{*}(\mu)\left(\sum_{1 \leq j \leq M} p_{i, j} \tilde{p}_{n c}(j)\right) .
\end{aligned}
$$

The above iterative procedure has been intensively used and validated by experiments [13].

\section{The Macrocell Residence Times}

This section derives the expected macrocell residence times to be used in the macrocell model in the next section. To simplify the description, we assume that the microcells are homogeneous (i.e., (12) and (17) hold) with the notations $\lambda_{o}=\lambda_{o}(i), \lambda_{h}=\lambda_{h}(i), p_{o}=p_{o}(i), p_{n c}=p_{n c}(i)$, $\eta=\eta_{i}, f_{m}(t)=f_{m, i}(t)$, and $r_{m}(t)=r_{m, i}(t)$. To accommodate heterogeneous microcells, the modifications on the model are straightforward but tedious.

In a homogeneous dual band GSM network, every radio coverage of a GSM900 BTS (a macrocell) overlays $N=$ $3 n^{2}-3 n+1$ DCS1800 BTSs (microcells) for some $n$ (in Fig. $2 \mathrm{a}, n=4)$. If a call cannot be served at a microcell, then it is switched to a macrocell. Since there are $N$ identical microcells in a macrocell, the overflow new call arrival rate to a macrocell is

$$
\lambda_{v o}=N p_{o} \lambda_{o} .
$$

Similarly, the overflow handoff call arrival rate to a macrocell is

$$
\lambda_{v h}=N p_{o} \lambda_{h} .
$$

Note that $p_{f}=p_{o}$ because the handoff calls and the new calls are indistinguishable from each other in GSM.

Suppose that an MS resides in a macrocell for a period $t_{M}$. During $t_{M}$, the MS visits $k$ microcells (i.e., the MS moves $k$ steps), and the MS resides at microcell $i$ for a period $t_{i}$. Then, $t_{M}=t_{1}+t_{2}+\ldots+t_{k}$ has the density function

$$
\begin{aligned}
f_{M}^{(k)}\left(t_{M}\right)= & \int_{t_{1}=0}^{t_{M}} \int_{t_{2}=0}^{t_{M}-t_{1}} \ldots \\
& \int_{t_{k-1}=0}^{t_{M}-t_{1}-\ldots-t_{k-2}} f_{m}\left(t_{1}\right) f_{m}\left(t_{2}\right) \ldots f_{m}\left(t_{k-1}\right) \\
& \times f_{m}\left(t_{M}-t_{1}-t_{2}-\ldots-t_{k-1}\right) d t_{k-1} \ldots d t_{2} d t_{1} .
\end{aligned}
$$

To accommodate a new/handoff call, an MS switches from a microcell to a macrocell if no channel is available in the microcell. Let $\tau_{M}$ be the period between when the MS switches to the macrocell and when the MS moves out of 


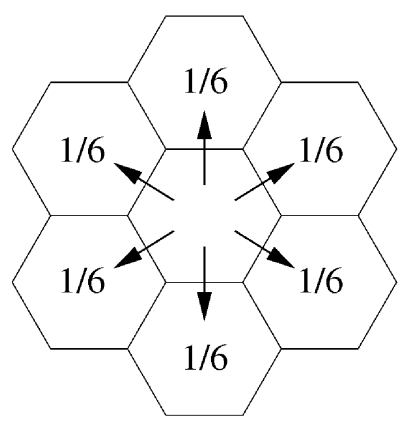

Fig. 4. The routing pattern.

the macrocell (see Fig. 3). Suppose that the MS visits $k$ microcells during $\tau_{M}$. Then, the density function of $\tau_{M}$ is

$$
\begin{aligned}
r_{M}^{(k)}\left(\tau_{M}\right)= & \left(\frac{\lambda_{v o}}{\lambda_{v o}+\lambda_{v h}}\right) \int_{\tau_{m}=0}^{\tau_{M}} \int_{t_{2}=0}^{\tau_{M}-\tau_{m}} \ldots \\
& \int_{t_{k-1}=0}^{\tau_{M}-\tau_{m}-t_{2}-\ldots-t_{k-2}} r_{m}\left(\tau_{m}\right) \\
& \times f_{m}\left(t_{2}\right) \ldots f_{m}\left(t_{k-1}\right) f_{m}\left(\tau_{M}-\tau_{m}-t_{2}-\ldots-t_{k-1}\right) \\
& d t_{k-1} \ldots d t_{2} d \tau_{m} \\
& +\left(\frac{\lambda_{v h}}{\lambda_{v o}+\lambda_{v h}}\right) f_{M}^{(k)}\left(\tau_{M}\right) .
\end{aligned}
$$

The first term of the righthand side in (21) represents the case that the MS switches from a microcell to a macrocell when a new call arrives. The second term of the righthand side in (21) represents the case that the MS switches from a microcell to a macrocell when a handoff call arrives.

Now, we describe a random walk model to compute when the MS leaves a macro cell. Our model considers a regular microcell/macrocell overlay structure shown in Fig. 2a. A macrocell is referred to as an $n$-layer macrocell if it overlays with $N=3 n^{2}-3 n+1$ microcells. Fig. 2a shows 4layer macrocells. The microcell at the center of the macrocell is called the layer 0 microcell. The microcells that surround layer $x-1$ microcells are called layer $x$ microcells. There are $6 x$ microcells in layer $x$ except exactly one microcell which is in layer 0 . An $n$-layer macrocell overlays microcells from layer 0 to layer $n-1$. The microcells that surround the layer $n-1$ microcells are referred to as boundary neighbors, which are outside of the macrocell.

We assume that an MS resides in a microcell for a period $t_{i}$, then moves to one of its neighbors with the same probability (i.e., with probability $1 / 6$; see Fig. 4 ). For an MS in conversation, we derive the number $k$ of moving steps (a step represents an MS movement from a microcell to another) from the beginning of the conversation until the MS moves out of the macrocell. A random walk model with $6(n-1)$ absorbing states in a two-dimensional hexagonal plan [5] can be used to study when an MS moves out of an $n$-layer macrocell boundary. In this model, a state represents the microcell where the MS resides. In order to compute the number of microcells visited before the MS leaves a macrocell, whenever the MS moves out of the macrocell, the random walk enters an absorbing state. A potential problem of this model is that the number of states increases rapidly as $n$ increases.

To reduce the computation complexity, we modify the two-dimensional random walk model based on our previous work [1]. According to the equal routing probability assumption, we classify the microcells in a macrocell into several microcell types. A microcell type is of the form $\langle x, y\rangle$, where " $x^{\prime \prime} \geq 0$ indicates that the microcell is in layer $x$ and $y \geq 0$ represents the $y+1$ st type in layer $x$. Microcells of the same type have the same traffic flow pattern because they are at the symmetrical positions on the hexagonal macrocell. Based on the derivation in [1], Fig. 5 illustrates the type of microcell for 6-layer macrocells. For example, the microcell in layer 0 is of the type $\langle 0,0\rangle$. The six microcells in layer 1 are grouped together and assigned to the same type $\langle 1,0\rangle$. A layer 2 microcell may have two or three neighbors in layer 3 and is assigned to type $\langle 2,1\rangle$ or $\langle 2,0\rangle$, respectively.

In the modified random walk model for an $n$-layer macrocell, a state $(x, y)$ represents that the MS is in one of the microcells of type $\langle x, y\rangle$, where $0 \leq x<n$ and $0 \leq y \leq x-1$. The state $(n, j)$ represents that the MS moves out of the macrocell from the state $(n-1, j)$, where $0 \leq j<n-1$. For $0 \leq x<n$ and $0 \leq y \leq x-1$, states $(x, y)$ are transient and, for $0 \leq j<n-1$, states $(n, j)$ are absorbing. The total number $S(n)$ of states for an $n$-layer macrocell random walk is $S(n)=\frac{n(n+1)}{2}$ and the computation complexity is significantly reduced. Details of the modified random walk and derivation of its steady state probabilities are given in [1] and the results are summarized as follows: The transition matrix of this random walk is an $S(n) \times S(n)$ matrix $P=\left(p_{(x, y),\left(x^{\prime}, y^{\prime}\right)}\right)$. An element $p_{(x, y),\left(x^{\prime}, y^{\prime}\right)}^{(k)}$ in $P^{(k)}$ is the probability that the random walk moves from state $(x, y)$ to state $\left(x^{\prime}, y^{\prime}\right)$ with exact $k$ steps. For $0 \leq j<n-1$, define $p_{k,(x, y),(n, j)}$ as

$$
p_{k,(x, y),(n, j)}= \begin{cases}p_{(x, y),(n, j)}, & \text { for } k=1, \\ p_{(x, y),(n, j)}^{(k)}-p_{(x, y),(n, j)}^{(k-1)}, & \text { for } k>1 .\end{cases}
$$

Then, $p_{k,(x, y),(n, j)}$ is the probability that an MS initially resides at a $\langle x, y\rangle$ microcell, moves into a $\langle n-1, j\rangle$ microcell at the $k-1$ st step and, then, moves out of the macrocell at the $k$ th step.

In a homogeneous dual band GSM network, an MS may be in any microcell of the overlaid macrocell with the same probability when it switches from the microcell to the macrocell during a phone call. On the other hand, the MS may enter a new macrocell through different types of microcells (and, thus, with different probabilities) during the conversation. Let $q_{(n-1, j)}$ be the probability that an MS enters the macrocell through a $\langle n-1, j\rangle$ microcell at the first step and $\tilde{q}_{(n-1, j)}$ be the probability that an MS moves out of the macrocell through a $\langle n-1, j\rangle$ microcell at the last step. Then, we have:

$$
\tilde{q}_{(n-1, j)}=\sum_{y=0}^{n-2} \sum_{k=1}^{\infty} q_{(n-1, y)} p_{k,(n-1, y),(n, j)} \quad \text { for } 0 \leq j<n-1
$$




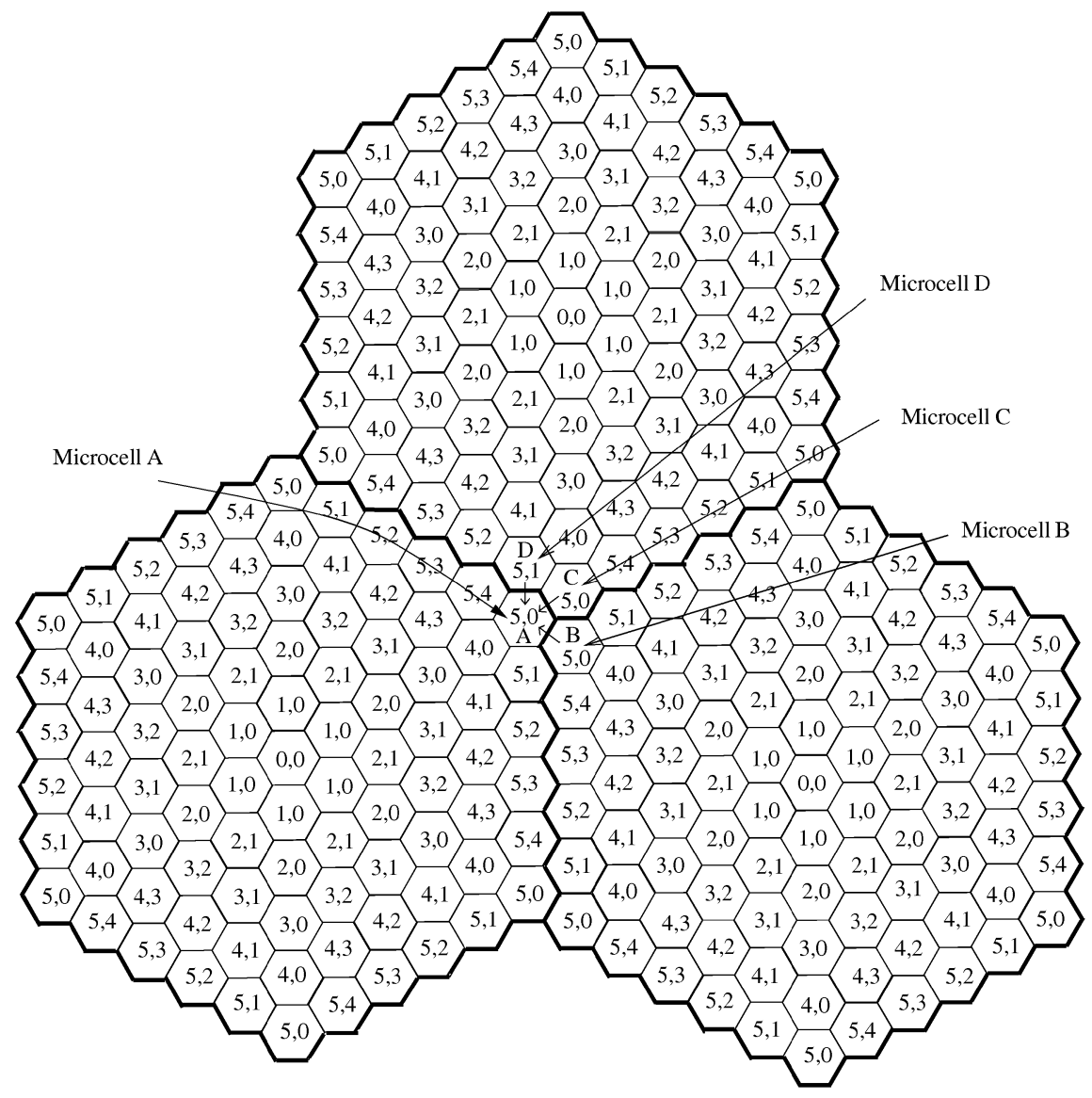

Fig. 5. Type assignments for 6-layer macrocells.

$$
\sum_{j=0}^{n-2} \tilde{q}_{(n-1, j)}=1
$$

Equation (22) indicates the situation where an MS moves out of the macrocell. Each term of the righthand side of (22) represents the probability that the MS initially resides at a $\langle n-1, y\rangle$ microcell and leaves the macrocell from a $\langle n-$ $1, j\rangle$ microcell at the $k$ th step. Equation (23) ensures that all probabilities sum up to 1 . For a 6-layer macrocell, we use $M_{2}=200$ truncated terms in (22) to approximate the infinite summations. Computation indicates that the error for the $p_{k,(n-1, y),(n, j)}$ summation truncation is within $10^{-4}$.

Now, we consider the situation where an MS passes through the boundary between two macrocells. For $n=2$,

$$
q_{(1,0)}=\tilde{q}_{(1,0)}=1 .
$$

For $n>2$, the following equations hold:

$$
\begin{gathered}
q_{(n-1,0)}=\frac{2}{3} \tilde{q}_{(n-1,0)}+\frac{1}{2} \tilde{q}_{(n-1,1)} \\
q_{(n-1,1)}=\frac{1}{3} \tilde{q}_{(n-1,0)}+\frac{1}{2} \tilde{q}_{(n-1, n-2)} \\
q_{(n-1, j)}=\frac{1}{2} \tilde{q}_{(n-1, n-j-1)}+\frac{1}{2} \tilde{q}_{(n-1, n-j)} \text { for } 2 \leq j<n-1 .
\end{gathered}
$$

We use the 6-layer macrocell configuration in Fig. 5 as an example to explain (24). The same reasoning applies to (25) and (26). Consider a $\langle 5,0\rangle$ microcell A. An MS entering microcell A comes from one of three surrounding boundary neighbors (microcell B, C or D). The probability that an MS at B moves out of the macrocell is $\tilde{q}_{(5,0)}$. Since B has three boundary neighbors, the probability that the MS moves from B to A is $\tilde{q}_{(5,0)} / 3$. Similarly, the MS moves from C to A with probability $\tilde{q}_{(5,0)} / 3$. For $0<j<n-1$, microcell $\langle 5, j\rangle$ only has two boundary neighbors. Thus, the MS moves from microcell $\mathrm{D}$ to microcell A with probability $\tilde{q}_{(5,1)} / 2$. Therefore, the righthand side of (24) indicates the probability that the MS moves to A from either B, C, or D. Following the same reasoning, (25) applies to microcell $\langle 5,1\rangle$ and (26) applies to microcells $\langle 5,2\rangle,\langle 5,3\rangle$, and $\langle 5,4\rangle$.

For $n=3$, (22)-(24) and (25) are used to compute $q_{(2,0)}$ and $q_{(2,1)}$. For $n>3$ and $0 \leq j<n-1$, we compute $q_{(n-1, j)}$ by using (22)-(26). For a 6-layer macrocell, we have $q_{(5,0)}=$ $27.27 \%$ and $q_{(5, j)}=18.18 \%$ for $1 \leq j \leq 4$.

From (20), the MS residence time density function for an $n$-layer macrocell is

$$
f_{M}\left(t_{M}\right)=\sum_{k=1}^{\infty} \sum_{y=0}^{n-2} \sum_{j=0}^{n-2} q_{(n-1, y)} p_{k,(n-1, y),(n, j)} f_{M}^{(k)}\left(t_{M}\right) \text { for } n>1 .
$$

From (20) and the Laplace transform convolution rule (P1.2.2 in [19]), the Laplace transform for $f_{M}^{(k)}(t)$ is 


$$
f_{M}^{(k) *}(s)=\left[f_{\mathbf{m}}^{*}(s)\right]^{k}
$$

and from (27), the Laplace transform for $f_{M}(t)$ is

$$
f_{\mathbf{M}}^{*}(s)=\sum_{k=1}^{\infty} \sum_{y=0}^{n-2} \sum_{j=0}^{n-2} q_{(n-1, y)} p_{k,(n-1, y),(n, j)}\left[f_{\mathbf{m}}^{*}(s)\right]^{k} \text { for } n>1 .
$$

From (21), the density function $r_{M}\left(\tau_{M}\right)$ for $\tau_{M}$ is

$$
\begin{aligned}
& r_{M}\left(\tau_{M}\right)=\sum_{k=1}^{\infty} \sum_{x=0}^{n-1} \sum_{y=0}^{x-1} \sum_{j=0}^{n-2}\left[\frac{\psi_{(x, y)}}{N} p_{k,(x, y),(n, j)} r_{M}^{(k)}\left(\tau_{M}\right)\right] \\
& \text { for } n>1,
\end{aligned}
$$

where $\psi_{\langle x, y\rangle}$ is the number of microcells of type $\langle x, y\rangle$ :

$$
\psi_{\langle x, y\rangle}= \begin{cases}1, & \text { for }\langle x, y\rangle=\langle 0,0\rangle \\ 6, & \text { otherwise }\end{cases}
$$

By using the Laplace transform convolution rule and from (7), (18), (19), and (21), we have

$$
r_{\mathbf{M}}^{(k) *}(s)=\left[\frac{\eta \lambda_{o}-\left(\eta \lambda_{o}-s \lambda_{h}\right) f_{\mathbf{m}}^{*}(s)}{s\left(\lambda_{o}+\lambda_{h}\right)}\right]\left[f_{\mathbf{m}}^{*}(s)\right]^{k-1} .
$$

For $n>1$ and from (29), the Laplace transform for $r_{M}(t)$ is

$$
\begin{array}{r}
r_{\mathbf{M}}^{*}(s)=\sum_{k=1}^{\infty}\left\{\left\{\sum_{x=0}^{n-1} \sum_{y=0}^{x-1} \sum_{j=0}^{n-2}\left[\frac{\psi_{(x, y)}}{N} p_{k,(x, y),(n, j)}\right]\right\}\right. \\
\left.\left[\frac{\eta \lambda_{o}-\left(\eta \lambda_{o}-s \lambda_{h}\right) f_{\mathbf{m}}^{*}(s)}{s\left(\lambda_{o}+\lambda_{h}\right)}\right]\left[f_{\mathbf{m}}^{*}(s)\right]^{k-1}\right\} .
\end{array}
$$

Let $t_{O N}$ and $t_{O H}$ be the channel occupation times of a macrocell for a new call (an overflow new/handoff call from a microcell) and a handoff call, respectively. From (13), (30), (14), and (28)

$$
\begin{aligned}
& E\left[t_{O N}\right]=\frac{1}{\mu}\left[1-r_{\mathrm{M}}^{*}(\mu)\right] \text { and } \\
& E\left[t_{O H}\right]=\frac{1}{\mu}\left[1-f_{\mathrm{M}}^{*}(\mu)\right] .
\end{aligned}
$$

Equations (28), (30), and (31) are computed with truncated terms. The Appendix shows how to select the number of truncated terms so that the errors for (28) and (30) can be controlled within a small predefined value.

\section{The Macrocell Model}

This section describes the macrocell model. The iterative algorithm for the macrocell model is similar to Algorithm 1.

\section{Algorithm 2 (Macrocell).}

\section{Input parameters}

- $\quad N=3 n^{2}-3 n+1$ : the number of microcells in an $n$ layer macrocell (given).

- $C$ : the number of channels in a macrocell (given).

- $1 / \mu$ : the expected call holding time (given).

- $\lambda_{v o}$ : the overflow new call arrival rate (computed by using (18), where $p_{o}$ is computed by Algorithm 1).
- $\lambda_{v h}$ : the overflow handoff call arrival rate (computed by using (19), where $p_{o}$ and $\lambda_{h}$ are computed by Algorithm 1).

- $\quad f_{\mathbf{M}}^{*}(s)$ : the Laplace transform of the $t_{M}$ distribution (derived by using (28)).

- $r_{\mathrm{M}}^{*}(s)$ : the Laplace transform of the density function for $\tau_{M}$ (derived by using (30)).

\section{Output measures}

- $\lambda_{H}$ : the handoff call arrival rate to a macrocell.

- $p_{O}$ : the new call blocking (handoff call forced termination) probability to a macrocell.

- $p_{n c, M}$ : the incompletion probability of a call (either blocked or forced to terminate) in the macrocell.

Step 1. Select an initial value for $\lambda_{H}$.

Step 2a. The load onto a macrocell is computed by using (32) and (33):

$$
\rho=\left(\lambda_{v o}+\lambda_{v h}\right) E\left[t_{O N}\right]+\lambda_{H} E\left[t_{O H}\right] .
$$

Step $\mathbf{2 b}$. The probability $p_{O}$ is computed by using the Erlang loss equation:

$$
p_{O}=B(\rho, C) .
$$

Step 3. $\lambda_{H, o l d} \leftarrow \lambda_{H}$.

Step 4. Compute $\lambda_{H}$ by using (12), (28), and (30):

$$
\lambda_{H}=\frac{\left(1-p_{O}\right) r_{\mathbf{M}}^{*}(\mu)\left(\lambda_{v o}+\lambda_{v h}\right)}{1-\left(1-p_{O}\right) f^{*}(\mu)} .
$$

Step 5. If $\left|\lambda_{H}-\lambda_{H, o l d}\right|>\delta \lambda_{H}$ (where $\delta$ is a predefined value), then go to Step 2. Otherwise, go to Step 6.

Step 6. The values for $\lambda_{H}$ and $p_{O}$ converge. Compute $p_{n c, M}$ by using (17), (28), and (30):

$$
p_{n c, M}=\frac{\left\{1+\left(1-p_{O}\right)\left[r_{\mathbf{M}}^{*}(\mu)-f_{\mathbf{M}}^{*}(\mu)\right]\right\} p_{O}}{1-\left(1-p_{O}\right) f_{\mathbf{M}}^{*}(\mu)} .
$$

Step 7. Compute the call incompletion probability as

$$
p_{N C}=p_{n c} p_{n c, M} .
$$

\section{Performance of the Dual Band PCS NETWORK}

We assume that the MS residence times in a microcell have a Gamma distribution with the shape parameter $\alpha$. As we mentioned before, the Gamma distribution is selected because it can be shaped to represent many distributions, as well as measured data that cannot be characterized by a particular distribution.

Fig. 6 shows the effect of the variation (or standard deviation) for the residence time distribution on $p_{N C}$. Note that, for Gamma distributions with the same mean value $1 / \eta$, the standard deviation $\sigma=\frac{1}{\eta \sqrt{\alpha}}$ increases as $\alpha$ decreases. By the Chebyshev's Inequality, the probability that the residence times are out of the range $[1 / \eta-5 \sigma / 3,1 / \eta+$ $5 \sigma / 3]$ is smaller than 36 percent for all $\sigma$ values. For 


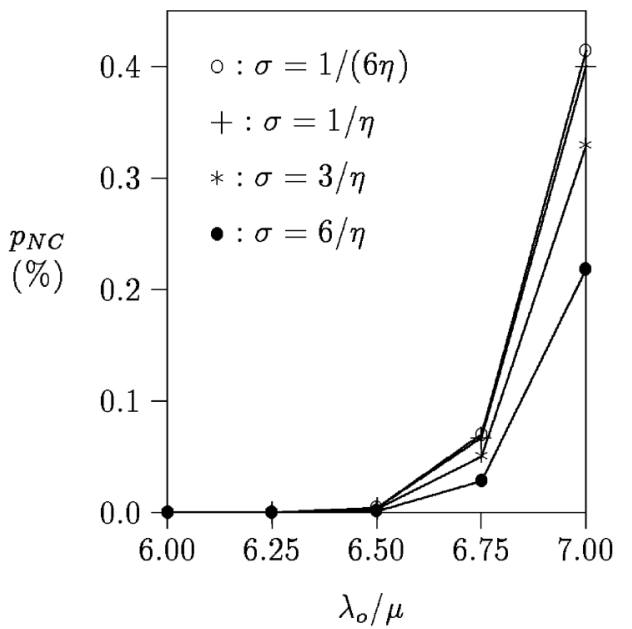

(a)

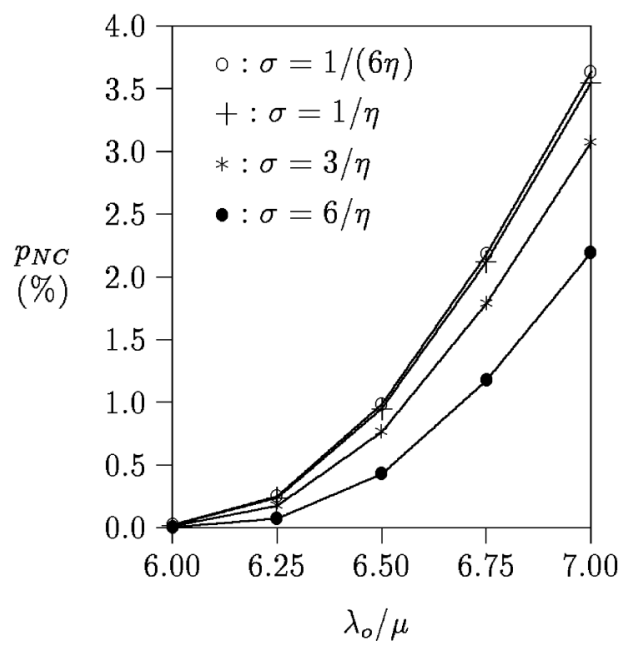

(c)

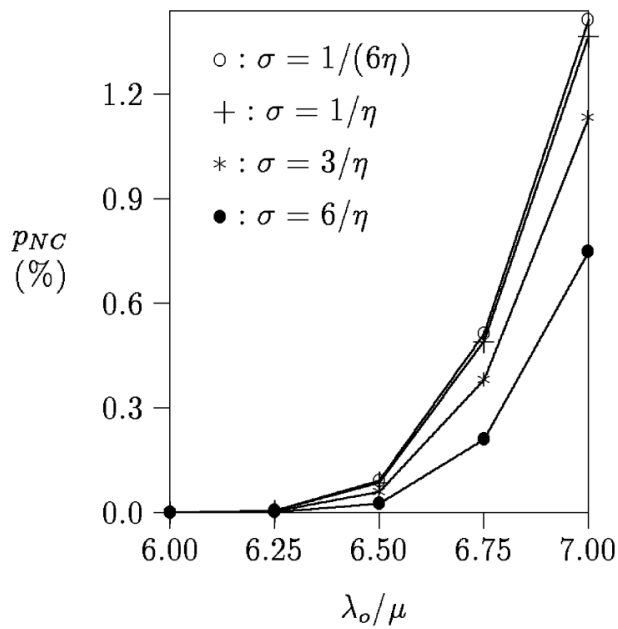

(b)

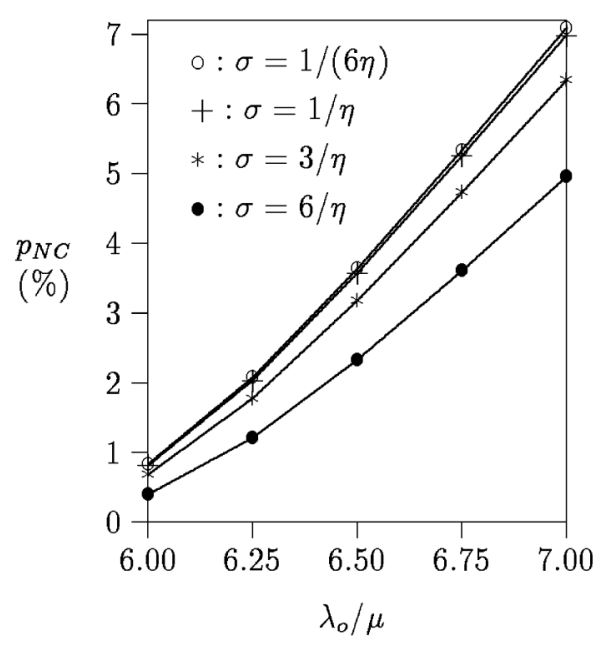

(d)

Fig. 6. The effect of the standard deviation for the microcell residence time distribution $(N=61, C=50, c=10)$. (a) $\eta=0.5 \mu$. (b) $\eta=\mu$. (c) $\eta=2 \mu$. (d) $\eta=4 \mu$.

example, if $\sigma=6 / \eta$, then $5 \sigma / 3=10 / \eta$ and the probability that the residence time exceeds $11 / \eta$ is smaller than 36 percent.

For a macrocell overlaying 61 microcells $(N=61)$ with 50 channels $(C=50)$, and the number of channels in each microcell is $10(c=10)$, we observe the following:

- $p_{N C}$ increases as $\sigma$ decreases.

- $\quad \sigma$ has a significant effect on $p_{N C}$ for $\sigma>1 / \eta$ and has almost no effect on $p_{N C}$ for $\sigma<1 / \eta$ (note that a Gamma distribution is exponential when $\sigma=1 / \eta$ ).

- $\quad p_{N C}$ increases as $\eta$ increases.

The call incompletion probability of the system increases as the standard deviation (variance) of the MS microcell residence time distribution decreases. For the same expected microcell residence times, more long and short residence times will be observed as the standard deviation increases. More long residence times implies that more new calls will complete without any handoff actions. Thus, smaller call incompletion probability is observed.

Figs. 7 and 8 illustrate the effect of the macrocell size. Four scenarios are considered: a macrocell of size $N=19$ with the channel number $C=16(C / N=0.8421), N=37$ with $C=31$ $(C / N=0.8378), N=61$ with $C=51(C / N=0.8361)$, and $N=91$ with $C=76(C / N=0.8352)$. We observe the following:

- For a fixed $C / N$ value, the performance of a large macrocell is better than that of a small macrocell.

- For a fixed $C / N$ value, the benefit of a large macrocell is significant for a large $\sigma$ value or a small $\eta$ value.

- For a fixed $C / N$ value, the benefit of a large macrocell becomes insignificant for heavy traffic.

Queuing theory indicates that systems with more common resource pool perform better than systems with isolated resources. Thus, the utilization of radio channels is more efficient for a large macrocell. Our study indicates that 


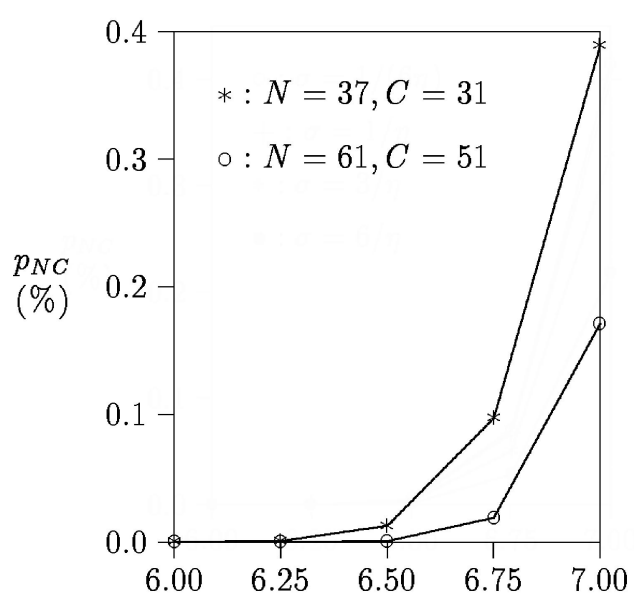

(a)

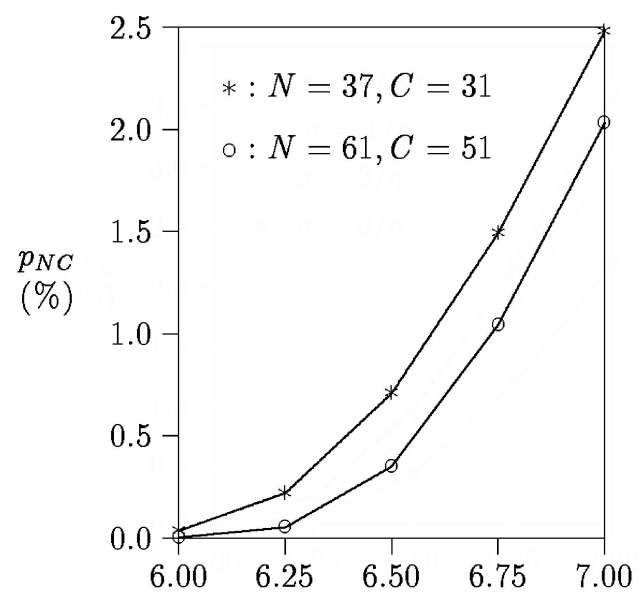

(c)

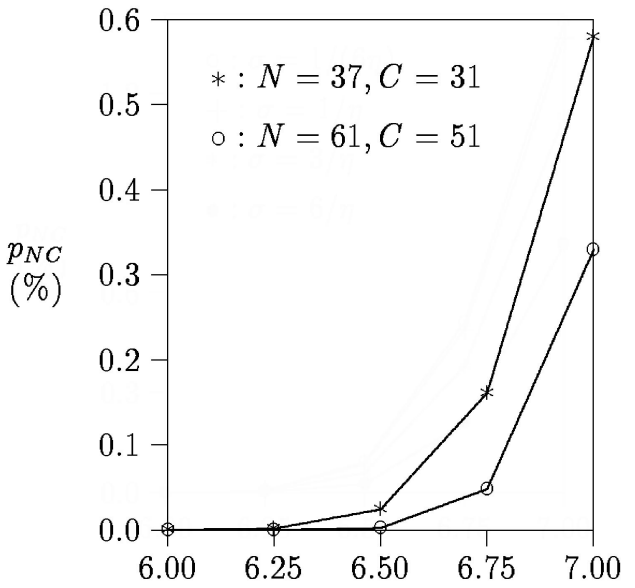

(b)

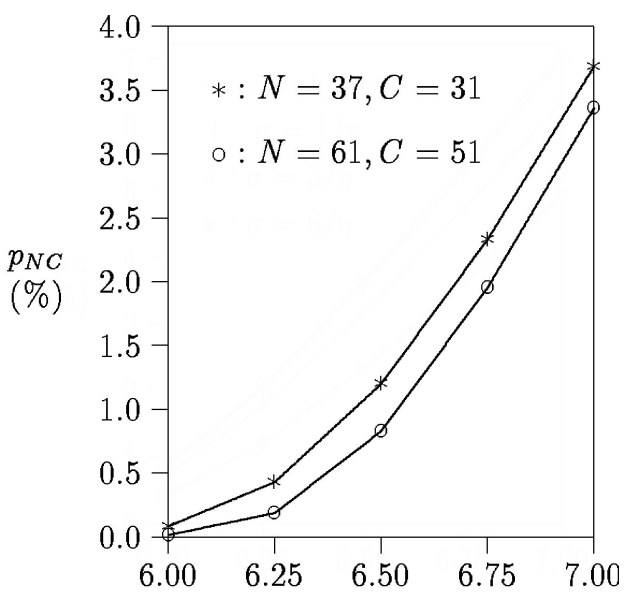

(d)

Fig. 7. The effect of the size of a macrocell $(c=10)$. (a) $\eta=0.5 \mu, \sigma=6 / \eta$. (b) $\eta=0.5 \mu, \sigma=1 / \eta$. (c) $\eta=2 \mu, \sigma=6 / \eta$. (d) $\eta=2 \mu, \sigma=1 / \eta$.

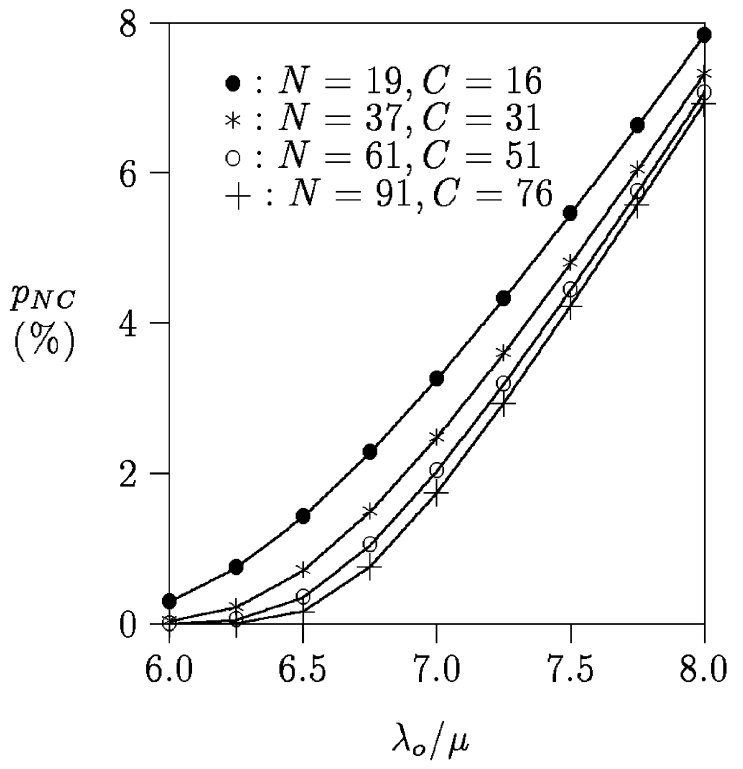

Fig. 8. The effect of the size of a macrocell $(c=10)$ for large traffic. $\eta=2 \mu, \sigma=6 / \eta$. large macrocells have good performance if $p_{N C}<2 \%$. About 7.05 offered load per microcell can be served by a macrocell with $N=91$ microcells and about 6.65 offered load per microcell can be served by a macrocell with $N=19$ microcells when $p_{N C}=2 \%$.

A single band PCS network is a special case of the dual band PCS network where $N=1$. Table 1 compares the performance of the single band system and the dual band system. In Table $1, N=61$ and $C=50$ for the dual band system. The table indicates that the dual band architecture significantly outperforms the single band system. For example, for $\sigma=1 /(6 \eta), \eta=\mu, \lambda_{o}=7 \mu, p_{N C}=1.413 \%$ for the dual band system with the total channels number $N \times c+C=660$. For the single band system, $p_{n c}=2.62 \%$, with the total channel number $61 \times 13=793$ or $p_{n c}=1.37 \%$ with the total channel number $61 \times 14=854$.

\section{Conclusions and Future Extensions}

We studied the performance of dual band GSM networks by assuming that a call is first served by a microcell. If no channel is available in the microcell, then the call is served 
TABLE 1

Comparison of the Single Band System and the Dual Band System $(N=61, C=50$, and $c=10$ for the Dual Band System

\begin{tabular}{|c|l|l|l|l|l|}
\hline New call arrival rate & $6 \mu$ & $6.25 \mu$ & $6.5 \mu$ & $6.75 \mu$ & $7 \mu$ \\
\hline$p_{N C}$ (Dual Band) & $0.000087 \%$ & $0.005402 \%$ & $0.090785 \%$ & $0.512134 \%$ & $1.412064 \%$ \\
\hline$p_{n c}$ (Single Band) & $0.000213 \%$ & $0.012760 \%$ & $0.144888 \%$ & $1.053400 \%$ & $2.622322 \%$ \\
& $(c=21)$ & $(c=18)$ & $(c=16)$ & $(c=14)$ & $(c=13)$ \\
& $0.000058 \%$ & $0.004199 \%$ & $0.055625 \%$ & $0.482846 \%$ & $1.352682 \%$ \\
& $(c=22)$ & $(c=19)$ & $(c=17)$ & $(c=15)$ & $(c=14)$ \\
\hline
\end{tabular}

(a)

\begin{tabular}{|c|l|l|l|l|l|}
\hline New call arrival rate & $6 \mu$ & $6.25 \mu$ & $6.5 \mu$ & $6.75 \mu$ & $7 \mu$ \\
\hline$p_{N C}$ (Dual Band) & $0.000011 \%$ & $0.000960 \%$ & $0.024639 \%$ & $0.209086 \%$ & $0.747114 \%$ \\
\hline$p_{n c}$ (Single Band) & $0.000015 \%$ & $0.001313 \%$ & $0.055557 \%$ & $0.477978 \%$ & $1.317863 \%$ \\
& $(c=23)$ & $(c=20)$ & $(c=17)$ & $(c=15)$ & $(c=14)$ \\
& $0.000004 \%$ & $0.000391 \%$ & $0.020114 \%$ & $0.204943 \%$ & $0.636520 \%$ \\
& $(c=24)$ & $(c=21)$ & $(c=18)$ & $(c=16)$ & $(c=15)$ \\
\hline
\end{tabular}

(b)

\begin{tabular}{|c|l|l|l|l|l|}
\hline New call arrival rate & $6 \mu$ & $6.25 \mu$ & $6.5 \mu$ & $6.75 \mu$ & $7 \mu$ \\
\hline$p_{N C}$ (Dual Band) & $0.003965 \%$ & $0.073178 \%$ & $0.428363 \%$ & $1.173599 \%$ & $2.192039 \%$ \\
\hline$p_{n c}$ (Single Band) & $0.011781 \%$ & $0.148035 \%$ & $0.516259 \%$ & $1.473158 \%$ & $3.412906 \%$ \\
& $(c=18)$ & $(c=16)$ & $(c=15)$ & $(c=14)$ & $(c=13)$ \\
& $0.003723 \%$ & $0.054869 \%$ & $0.214707 \%$ & $0.698493 \%$ & $1.861521 \%$ \\
& $(c=19)$ & $(c=17)$ & $(c=16)$ & $(c=15)$ & $(c=14)$ \\
\hline
\end{tabular}

(c)

(a) $\sigma=1 /(6 \eta), \eta=\mu$. (b) $\sigma=6 / \eta, \eta=\mu$. (c) $\sigma=6 / \eta, \eta=2 \mu$.

by the overlaid macrocell. The call is blocked (or forced to terminate) if no channel is available in the macrocell. We observed the following:

- The call incompletion probability of the system increases as the standard deviation of the MS microcell residence time distribution decreases. Furthermore, the call incompletion probability of the system increases as the user mobility increases, though the forced termination probability is decreased. These results are consistent with those in the single band system [13].

- For the same channel budget, the call incompletion probability decreases as the number $N$ of microcells covered by a macrocell increases. However, the size of a macrocell cannot be arbitrarily increased due to power consumption of the radio system. The tradeoff between the size of $N$ and the power consumption deserves further study.

This paper assumed that the switching of a call from a microcell to a macrocell is based on the channel availability of the microcell (the model in [7] followed the same assumption). However, a call may be switched according to the mobility of a mobile user as suggested in [8]. If a mobile user moves too fast, the calls may be served by macrocells to reduce the number of handoffs. Our model can be easily modified to accommodate this condition as follows:

- Suppose that the user's speed can be measured. For example, we define the user moves too fast if 5 microcells have been visited during a fixed period of three minutes. Compute the probability $\beta$ that a mobile user is too fast.

- In Fig. $2 b, 1-\beta$ of the handoff/new call traffic (i.e., the portion of the call traffic generated by the slow moving users) is directed to the microcell models as before and $\beta$ of the traffic (i.e., the portion of the traffic generated by the fast moving users) is directed to the overlaying macrocell.

\section{APPENDIX}

\section{Selecting the Number of Truncated Terms for $E\left[t_{O N S}\right]$ AND $E\left[t_{O H S}\right]$}

The summations of infinite series in (30) and (28) can be

accurately approximated by the summations of finite series

as follows: Note that $0<f_{\mathbf{M}}^{*}(\mu)<1$. For (30), let $s=\mu$,

$$
X=\frac{\eta \lambda_{o}-\left(\eta \lambda_{o}-\mu \lambda_{h}\right) f_{\mathbf{m}}^{*}(\mu)}{\mu\left(\lambda_{o}+\lambda_{h}\right)}>0
$$

and note that

$$
0 \leq Y=\sum_{x=0}^{n-1} \sum_{y=0}^{x-1} \sum_{j=0}^{n-2} \frac{\psi_{(x, y)}}{N} p_{k,(x, y),(n, j)} \leq 1 .
$$

Consider a positive value $\epsilon<<1$ and an integer $K_{1}$. We

have

$$
\begin{aligned}
K_{1} \geq\left\lceil\frac{\log \left\{\frac{\epsilon}{X}\left[1-f_{\mathbf{m}}^{*}(\mu)\right]\right\}}{\log \left[f_{\mathbf{m}}^{*}(\mu)\right]}\right] & \Rightarrow \sum_{k=K_{1}+1}^{\infty} X\left[f_{\mathbf{m}}^{*}(\mu)\right]^{k-1} \leq \epsilon \\
& \Rightarrow \sum_{k=K_{1}+1}^{\infty} X Y\left[f_{\mathbf{m}}^{*}(\mu)\right]^{k-1} \leq \epsilon
\end{aligned}
$$

Similarly, for an integer $K_{2}$ 


$$
\begin{aligned}
& K_{2} \geq\left\lceil\frac{\log \left\{\epsilon\left[1-f_{\mathbf{m}}^{*}(\mu)\right]\right\}}{\log \left[f_{\mathbf{m}}^{*}(\mu)\right]}\right\rceil-1 \\
\Rightarrow & \sum_{k=K_{2}+1}^{\infty} \sum_{y=0}^{n-2} \sum_{j=0}^{n-2} q_{(n-1, y)} p_{k,(n-1, y),(n, j)}\left[f_{\mathbf{m}}^{*}(\mu)\right]^{k} \leq \epsilon .
\end{aligned}
$$

Let $K=\max \left(K_{1}, K_{2}\right)$, then the error introduced by the following approximations for $E\left[t_{O N}\right]$ and $E\left[t_{O H}\right]$ is bounded by $\epsilon / \mu$ :

$$
\begin{gathered}
E\left[t_{O N}\right]=\frac{1}{\mu}-\frac{1}{\mu}\left\{\sum _ { k = 1 } ^ { K } \left\{\left[\sum_{x=0}^{n-1} \sum_{y=0}^{x-1} \sum_{j=0}^{n-2} \frac{\psi_{(x, y)}}{N} p_{k,(x, y),(n, j)}\right]\right.\right. \\
\left.\left.\left[\frac{\eta \lambda_{o}-\left(\eta \lambda_{o}-\mu \lambda_{h}\right) f_{\mathbf{m}}^{*}(\mu)}{\mu\left(\lambda_{o}+\lambda_{h}\right)}\right]\left[f_{\mathbf{m}}^{*}(\mu)\right]^{k-1}\right\}\right\} .
\end{gathered}
$$

and

$$
E\left[t_{O H}\right]=\frac{1}{\mu}\left[1-\sum_{k=1}^{K} \sum_{y=0}^{n-2} \sum_{j=0}^{n-2} q_{(n-1, y)} p_{k,(n-1, y),(n, j)}\left[f_{\mathbf{m}}^{*}(\mu)\right]^{k}\right] .
$$

\section{References}

[1] I.F. Akyildiz, Y.-B. Lin, W.-R. Lai, and R.-J. Chen, "A New Model for Random Walks in PCS Networks," Submitted for publication, Technical Report CSIE-PCS2000-1; see http://liny.csie.nctu. edu.tw, 1999.

[2] R. Beraldi, S. Marano, and C. Mastroianni, "Performance of a Reversible Hierarchical Cellular System," Int'l J. Wireless Information Networks, vol. 4, no. 1, pp. 43-54, 1997.

[3] Y. Fang, I. Chlamtac, and Y.-B. Lin, "Channel Occupancy Times and Handoff Rate for Mobile Computing and PCS Networks," IEEE Trans. Computers, vol. 47, no. 6, pp. 679-692, June 1998.

[4] Y. Fang, I. Chlamtac, and Y.-B. Lin, "Modeling PCS Networks under General Call Holding Time and Cell Residence Time Distributions," IEEE/ACM Trans. Networking, vol. 5, no. 6, pp. 893906, 1998.

[5] W. Feller, An Introduction to Probability Theory and Its Applications, Vol. I. John Wiley \& Sons, 1966.

[6] G.J. Foschini, B. Gopinath, and Z. Miljanic, "Channel Cost of Mobility," IEEE Trans. Vehicular Technology, vol. 42, no. 4, pp. 414424, Nov. 1993.

[7] L.R. Hu and S.S. Rappaport, "Micro-Cellular Communication Systems with Hierarchical Macrocell Overlays: Traffic Performance Models and Analysis," Proc. WINLAB Workshop, pp. 143174, 1993.

[8] C.L. I?, L.J. Greenstein, and R.D. Gitlin, "A Microcell/Macrocell Cellular Architecture for Low- and High-Mobility Wireless Users," IEEE J. Selected Areas in Comm., vol. 11, no. 6, pp. 885891, 1993.

[9] N.L. Johnson, Continuous Univariate Distributions-1. John Wiley \& Sons, 1970.

[10] I. Katzela and M. Naghshineh, "Channel Assignment Schemes for Cellular Mobile Telecommunication Systems: A Comprehensive Survey," IEEE Personal Comm., vol. 3, no. 3, pp. 10-31, June 1996.

[11] F.P. Kelly, Reversibility and Stochastic Networks. John Wiley \& Sons, 1979.

[12] L. Kleinrock, Queueing Systems: Vol. I-Theory. New York: Wiley, 1976.

[13] Y.-B. Lin, "Performance Modeling for Mobile Telephone Networks," IEEE Network Magazine, vol. 11, no. 6, pp. 63-68, Nov./ Dec. 1997.

[14] Y.-B. Lin, L.-F. Chang, and A. Noerpal, "Modeling Hierarchical Microcell/Macrocell PCS Architecture," Proc. IEEE Int'l Conf. Comm., 1995.

[15] Y.-B. Lin, S. Mohan, and A. Noerpel, "Queueing Priority Channel Assignment Strategies for Handoff and Initial Access for a PCS Network," IEEE Trans. Vehicular Technology, vol. 43, no. 3, pp. 704712, 1994.

[16] M. Mouly and M.-B. Pautet, The GSM System for Mobile Communications. 1992.
[17] S. Tekinary and B. Jabbari, "A Measurement Based Prioritization Scheme for Handovers in Cellular and Microcellular Networks," IEEE J. Selected Areas in Comm., pp. 1,343-1,350, Oct. 1992.

[18] L.-C. Wang, G.L. Stüber, and C.-T. Lea, "Architecture Design, Frequency Planning, and Performance Analysis for a Microcell/ Macrocell Overlaying System," IEEE Trans. Vehicular Technology, vol. 46, no. 4, pp. 836-848, 1997.

[19] E.J. Watson, Laplace Transforms and Applications. Birkhauserk, 1981.

[20] M.D. Yacoub, Foundations of Mobile Radio Engineering. CRC Press, 1993.

[21] C.H. Yoon and K. Un, "Performance of Personal Portable Radio Telephone Systems with and without Guard Channels," IEEE J. Selected Areas in Comm., vol. 11, no. 6, pp. 911-917, Aug. 1993

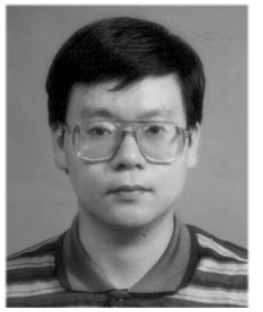

Yi-Bing Lin (S'80-M'96-SM'96) received his BSEE degree from National Cheng Kung University in 1983 and his $\mathrm{PhD}$ degree in computer science from the University of Washington in 1990. From 1990 to 1995, he was with the Applied Research Area at Bell Communications Research (Bellcore), Morristown, New Jersey. In 1995, he was appointed a professor in the Department of Computer Science and Information Engineering (CSIE), National Chiao Tung University (NCTU). In 1996, he was appointed deputy director of the Microelectronics and Information Systems Research Center, NCTU. During 1997-1999, he was elected chairman of CSIE, NCTU. His current research interests include design and analysis of personal communications services network, mobile computing, distributed simulation, and performance modeling.

Dr. Lin is an associate editor of IEEE Network, an editor of the IEEE Journal of Selected Areas in Communications: Wireless Series, IEEE Personal Communications Magazine, Computer Networks, ACM Mobile Computing and Communication Review, International Journal of Communications Systems, ACM/Baltzer Wireless Networks, Computer Simulation Modeling and Analysis, Journal of Information Science and Engineering, a columnist for ACM Simulation Digest, and guest editor for the $A C M / B a l t z e r$ MONET special issue on personal communications, IEEE Transactions on Computers special issue on mobile computing, and IEEE Communications Magazine special issue on active, programmable, and mobile code networking. Dr. Lin received the 1997 Outstanding Research Award from the National Science Council, Republic of China (ROC), and the Outstanding Youth Electrical Engineer Award from CIEE, ROC.

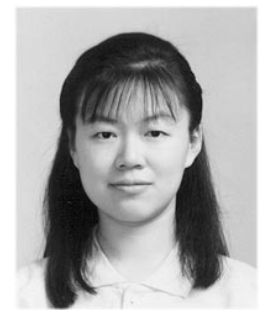

Wei-Ru Lai received her BSEE degree and $\mathrm{PhD}$ degrees from the Department of Computer Science and Information Engineering, National Chiao Tung University in 1991 and 1999, respectively. In 1999, she was appointed an assistant professor and was elected chairman of the Department of Information Management, Chin-Min College. Her current research interests include design and analysis of personal communications services network.

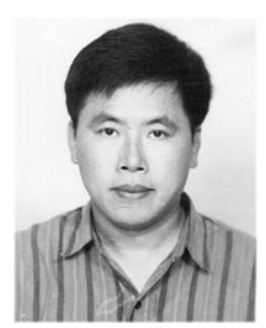

Rong-Jaye Chen received his BS (1977) in mathematics from National Tsing Hua University and his $\mathrm{PhD}$ (1987) in computer science from the University of Wisconsin-Madison. $\mathrm{He}$ is currently a professor and chairman of Computer Science and Information Engineering Department at National Chiao Tung University. His research interests include cryptography and security, personal communication service, algorithm design, and theory of computation. 